American Journal of Applied Sciences 3 (6): 1899-1902, 2006

ISSN 1546-9239

(c) 2006 Science Publications

\title{
Satellite's Motion under the Effect of Magnetic Torque
}

\author{
Rashmi Bhardwaj and Parsan Kaur \\ Department of Mathematics, School of Basic and Applied Sciences \\ Guru Gobind Singh Indraprastha University, Kashmere Gate, Delhi-110006, India
}

\begin{abstract}
Non-integrability of planar oscillation of a satellite in an elliptic orbit under the influence of magnetic torque has been studied. The amplitude of the oscillation remains constant upto the second order of approximation. The analysis regarding the stability of the stationary planar oscillation of a satellite near the resonance frequency shows that discontinuity occurs in the amplitude of the oscillation at a frequency of the external periodic force which is less than the frequency of the natural oscillation.
\end{abstract}

Key words: Non-linear, resonance, non-resonance, magnetic torque

\section{INTRODUCTION}

Maciejewski $^{[1]}$, Bhardwaj ${ }^{[2]}$, Schweighart et al. ${ }^{[3]}$ discussed the non-integrability of a rotational motion of a rigid satellite under different torques. Suli ${ }^{[4]}$, Michtchenko et.al. ${ }^{[5]}$, Murray et.al. ${ }^{[6]}$, Kotoulas et al..$^{[7]}$, Hadjidemetriou $^{[8]}$, studied the resonance in Solar system.

\section{EQUATION OF MOTION}

The Satellite S moving in an elliptic orbit around the Earth E such that the orbital plane coincides with the equatorial plane of the Earth (Fig.1.) with principal moments of inertia $\mathrm{A}<\mathrm{B}<\mathrm{C}$ at its centre of mass. Let $\vec{r}$ be the radius vector of the center of mass of the satellite, $\theta$ the angle that the long axis of the satellite make with a fixed line EF lying in the orbital plane, $v$ true anomaly and $\eta=\delta=q$ the angle between the radius vector and the long axis. The instantaneous magnetic disturbance torque, $[\bar{N}$ in N.m] due to the spacecraft effective magnetic moment $\bar{m}$ (in A. $\mathrm{m}^{2}$ ) is given by $\bar{N}=\bar{m} \times \bar{B}, \quad \bar{B} \quad$ is the geocentric magnetic flux density (in $\mathrm{Wb} / \mathrm{m}^{2}$ ) and $\bar{m}$ is the sum of the individual magnetic moments caused by permanent and induced magnetism and the spacecraft generated current loops. Euler's dynamical equation of motion in elliptic orbit about z-axis is

$$
\begin{aligned}
& (1+e \cos v) \frac{d^{2} \eta}{d v^{2}}-2 e \sin v \frac{d \eta}{d v}- \\
& -4 e \sin v+n^{2} \sin \eta=\varepsilon \sin \left(a_{1}+b v\right)
\end{aligned}
$$

where, $n^{2}=\frac{3(B-A)}{C}, \quad \varepsilon=\frac{3 a^{3} m H_{o}}{\mu C} \sin ^{2} \theta_{m}^{\prime}=$ parameter due to magnetic torque, $\Omega_{1}$ is angular velocity of the satellite and $\mathrm{e}$ is the eccentricity of the orbit of a satellite,

$a_{1}=2\left(\Omega_{0}-\alpha_{G o}-\phi_{m}^{\prime}\right), \quad b=2\left(1+\frac{1}{\Omega_{1}}\left(\frac{d \Omega}{d t}-\frac{d \alpha_{G}}{d t}\right)\right), \quad a^{3} H_{0}=$ total dipole strength, $\alpha_{G o}=$ right ascension of the Greenwich meridian at some reference time, $\frac{d \alpha_{G}}{d t}=$ average rotation rate of the earth, $\theta_{m}^{\prime}=$ coelevation of the dipole, $\phi_{m}^{\prime}=$ east longitude of the dipole, $\frac{d \Omega}{d t}=-2.06474 \times 10^{14} \frac{a^{-7 / 2} \cos i}{\left(1-e^{2}\right)^{2}}=0.9856^{\circ} /$ day.,$\Omega_{0}=210^{\circ}$, $\mu=G\left(m_{E}+m_{S}\right)=G m_{E}, \quad m_{E}=$ mass of the Earth, $m_{S}=$ mass of the satellite.

Equation (1) is equivalent to the Hamilton's equation $\frac{d q}{d v}=\frac{\partial H}{\partial p}, \frac{d p}{d v}=-\frac{\partial H}{\partial q}$, where,

$H=H_{o}+\varepsilon H_{1}+\ldots \ldots . . ., H_{o}=\frac{p^{2}}{2}-2 p-n^{2} \cos q$,

$H_{1}=-p^{2} \cos v-n^{2} \cos q \cos v-\varepsilon_{1} \sin (a+b v) q \mathrm{p}=$ generaliz ed momenta.

The hyperbolic equilibrium solution corresponding to $\mathrm{H}_{0}$ is given by $p(v)=2, q(v)=0, \Pi, \ldots \ldots$. , The unperturbed double asymptotic solutions are given by

$$
\begin{aligned}
& p^{ \pm}(v)=2 \pm \frac{2 n}{\cosh n v}, \quad \quad \sin \left(q^{ \pm}(v)\right)= \pm \frac{2 \sinh (n v)}{\cosh ^{2}(n v)}, \\
& \cos \left(q^{ \pm}(v)\right)=\frac{2}{\cosh ^{2}(n v)}-1 .
\end{aligned}
$$

\section{EVALUATION OF MELNIKOV'S INTEGRAL}

Corresponding Author: Dr. Rashmi Bhardwaj, Department of Mathematics, School of Basic and Applied Sciences, Guru Gobind Singh Indraprastha University, Kashmere Gate, Delhi - 110006, India 1899 
Melnikov function,

$$
\begin{aligned}
& M^{ \pm}\left(v_{0}\right)=\int_{-\infty}^{\infty}\left(H_{0}, H_{1}\right)\left(q^{ \pm}\left(v-v_{0}\right), p^{ \pm}\left(v-v_{0}\right)\right) d v \\
& = \pm 8 \pi \sin \left(v_{0}\right) \sec h\left(\frac{\pi}{2 n}\right) \pm 6 \pi \sin \left(v_{0}\right) \operatorname{cosech}\left(\frac{\pi}{2 n}\right) \\
& \pm 2 \varepsilon_{1} \pi \sin \left(a_{1}+b v_{0}\right) \sec h\left(\frac{\pi b}{2 n}\right)
\end{aligned}
$$

It is easy to observe that for any value of mass parameter $\mathrm{n}$ and magnetic torque parameters $\varepsilon_{1}$ and $a_{1}\left(0.107125 \leq a_{1} \leq 0.220081\right)$ the above function has a simple zero. Thus, both pairs of asymptotic surfaces cross transversely and eqn. (1) is non-integrable.

\section{GRAPHICAL REPRESENTATION OF MELNIKOV'S FUNCTION}

In Earth-Artificial Satellite System with data as: $\Omega_{1}=\frac{2 \Pi}{30 \times 24 \times 60 \times 60} \mathrm{rad} / \mathrm{s}, \mathrm{e}=0.0549, \varepsilon=0.001, \varepsilon_{1}$ $=0.018214936$ (as $\mathcal{E}=\mathrm{e} \varepsilon_{1}$ ) and $\mathrm{b}=2.164264351851$.

1. In Fig. 2, for fixed value of $b=2.164264351851$, $a_{1}=0.10916685405$ and for $0 \leq v_{0} \leq 12$ the Melnikov's function behaves almost like sine functions with abscissas almost remain same and as $\mathrm{n}$ vary from 0.1 to $0.12, \mathrm{M}^{+}\left(\mathrm{v}_{0}\right)$ and $\mathrm{M}^{-}\left(\mathrm{v}_{0}\right)$ elongate along the ordinate.

2. In Fig. 3, for fixed value of $b=2.164264351851$, $a_{1}=0.10916685405, \mathrm{v}_{0}=0.1$ and for $0.1 \leq \mathrm{n} \leq 0.99$, as mass distribution changes, the value of Melnikov's function $\quad M^{ \pm}\left(v_{0}, n, a_{1}\right)$ increases/decreases very slowly for $0.1 \leq \mathrm{n} \leq 0.3$ and after that it increases/decreases exponentially.

3. In Fig. 4 for fixed value of $b=2.164264351851$, $\mathrm{v}_{0}=0.1, \mathrm{n}=0.1$ and for $0.107125 \leq a_{1} \leq 0.220081$, as $a_{1}$ due to magnetic torque effect changes, the value of Melnikov's function $M^{+}\left(v_{0}, n, a\right)$ remains almost constant to 0.00000132343 . These values of $a_{1}$ are tabulated in Table 1 .

\section{NON RESONANT MOTION OF SATELLITE}

Taking $\mathrm{n}^{2}=\alpha$ e and $\varepsilon=e \varepsilon_{1}$, eqn. (1) becomes

$$
\frac{d^{2} \eta}{d v^{2}}+n^{2} \eta=e f\left(v, \eta, \frac{d \eta}{d v}, \frac{d^{2} \eta}{d v^{2}}\right)
$$

where,

$$
\begin{gathered}
f\left(v, \eta, \eta^{\prime}, \eta^{\prime \prime}\right)=4 \sin v+2(\sin v) \eta^{\prime}- \\
(\cos v) \eta^{\prime \prime}+\alpha(\eta-\sin \eta)+\varepsilon_{1} \sin \left(a_{1}+b v\right)
\end{gathered}
$$

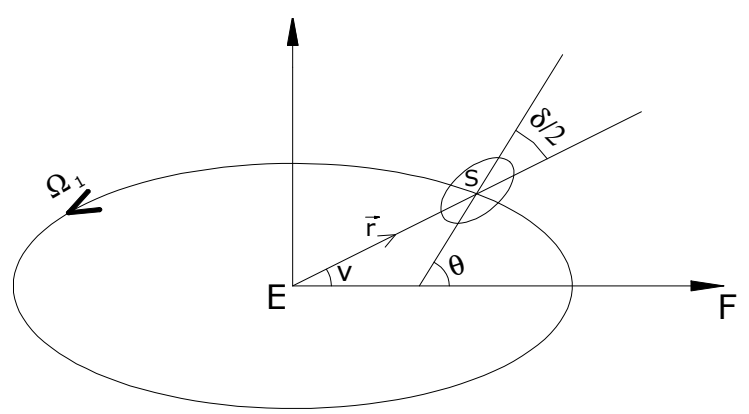

Fig. 1: Satellite planar oscillation in elliptic orbit with magnetic perturbation

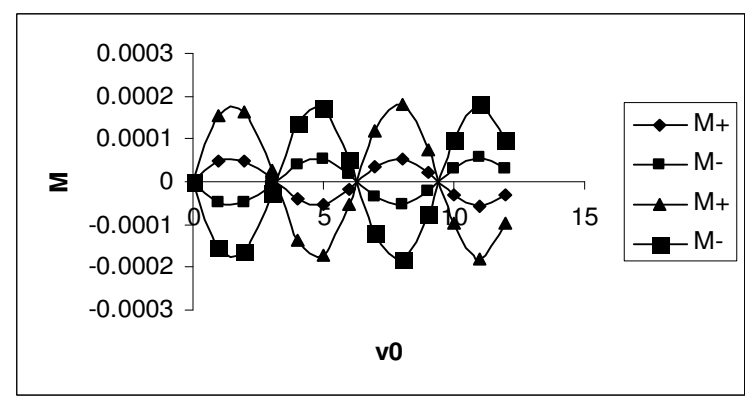

Fig. 2: Melnikov's function $\quad M^{ \pm}\left(v_{0}, n, a_{1}\right)$ for $0 \leq v_{0} \leq 12, a_{1}=0.10916685405, \mathrm{n}=0.11,0.12$

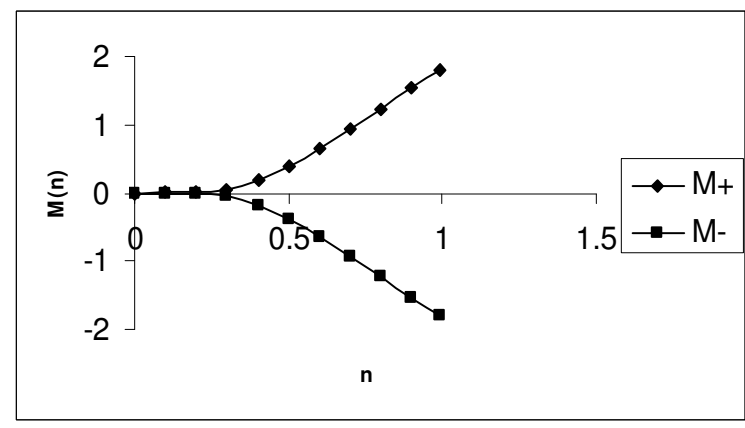

Fig. 3: Melnikov's function $M^{ \pm}\left(v_{0}, n, a_{1}\right)$ for 0.1 $\leq \mathrm{n} \leq 0.99 \mathrm{v}_{0}=0.1, a_{1}=0.10916685405$

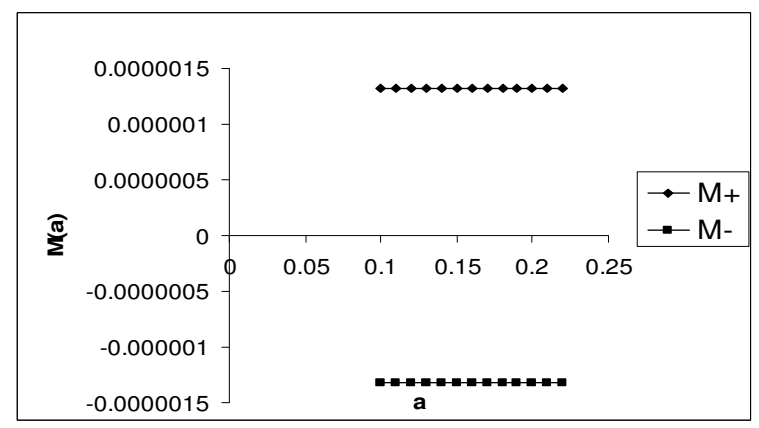

Fig. 4: Melnikov's function $M^{ \pm}\left(v_{0}, n, a_{1}\right)$ for $\mathrm{v}_{0}=0.1, \mathrm{n}=0.1,0.107125 \leq a_{1} \leq 0.220081$ 
Table 1: Values of $a$ from 2001 to 2050 at $0^{\mathrm{h}} \mathrm{UT}$ December 31

\begin{tabular}{llllc}
\hline S.No. & Year & $\alpha_{\mathrm{G} 0}$ (in deg) & $\varphi_{\mathrm{m}}{ }^{\prime}$ (in deg) & $a_{1}$ (in Radians) \\
\hline 1 & 2001 & 98.9429 & 107.988 & 0.10712 \\
2 & 2009 & 98.9829 & 107.472 & 0.12374 \\
3 & 2017 & 99.0229 & 106.936 & 0.14103 \\
4 & 2025 & 99.0629 & 106.381 & 0.15900 \\
5 & 2033 & 99.1029 & 105.805 & 0.17771 \\
6 & 2041 & 99.1429 & 105.207 & 0.19719 \\
7 & 2050 & 99.1879 & 104.507 & 0.22008 \\
\hline
\end{tabular}

The dynamical system described by eqn. (3) moves under forced vibrations due to the presence of the periodic sine forces on the right hand side of the equation.

In the first approximation, thus, using BKM method (Bhardwaj[1]), the solution is given by $\eta=a \cos \psi$, where the amplitude $a$ and the phase $\psi$ are given by $\frac{d a}{d v}=0 \Rightarrow a=$ constant, $\frac{d \psi}{d v}=n+\frac{n}{2 a}\left(2 J_{1}(a)-a\right)$ and in the second approximation, the solution is obtained as

$\eta=a \cos \psi+e\left(\begin{array}{c}\frac{4 \sin v}{n^{2}-1}-\left(n a+\frac{n^{2} a}{2}\right) \frac{\cos (v+\psi)}{2 n+1}+\left(\frac{n^{2} a}{2}-n a\right) \frac{\cos (v-\psi)}{2 n-1} \\ +\frac{\varepsilon_{1} \sin \left(a_{1}+b v\right)}{n^{2}-b^{2}}+\frac{\alpha}{2 n^{2}} \sum_{k=1}^{\infty} \frac{(-1)^{k} J_{2 k+1}(a) \cos (2 k+1) \psi}{k(k+1)}\end{array}\right)$

where the amplitude $a$ and the phase $\psi$ are given by $\frac{d a}{d v}=0 \Rightarrow a=$ constant,

$$
\begin{aligned}
& \frac{d \psi}{d v}=n+\frac{n}{2 a}\left(2 J_{1}(a)-a\right)+\frac{n}{2 a} \sum_{k=1}^{\infty} \frac{J_{2 k+1}(a) J_{2 k+1}^{\prime}(a)}{k(k+1)} \\
& -\frac{n}{8 a^{2}}\left(2 J_{1}(a)-a\right)^{2}+e^{2} \frac{3 n\left(n^{2}-1\right)}{4\left(4 n^{2}-1\right)}
\end{aligned}
$$

The amplitude of the oscillation remains constant upto the second order of approximation and the main resonance occurs at $n \cong b$ and $n \cong 1$ and the parametric resonance occurs for $n \cong \frac{1}{2}$.

\section{RESONANT PLANAR OSCILLATIONS OF A SATELLITE}

Using BKM method for resonances at $\mathrm{n}=1$, equating the coefficients of $\mathrm{e}$ and using Fourier expansion in terms of Bessel's function in eqn (3), we get

$$
\left((n-1) \frac{\partial A_{1}}{\partial \theta}-2 a n B_{1}\right) \cos \psi-\left((n-1) a \frac{\partial B_{1}}{\partial \theta}+2 n A_{1}\right)
$$$$
\sin \psi=4 \sin (\psi-\theta)-2 \text { an } \sin (\psi-\theta) \sin \psi
$$$$
+a n^{2} \cos (\psi-\theta) \cos \psi+\alpha\left(a-2 J_{1}(a)\right) \cos \psi-
$$

$2 \alpha \sum_{j=1}^{\infty}(-1)^{j} J_{2 j+1}(a) \cos (2 j+1) \psi+\varepsilon_{1} \sin (a+b \psi-b \theta)$

Comparing the coefficients of like powers of $\cos (\psi)$ and $\sin (\psi)$ to zero and solving, we get

$A_{1}=\frac{-4}{n+1} \cos \theta, \quad B_{1}=\frac{4}{a(n+1)} \sin \theta-\frac{\alpha}{2 a n}\left(a-2 J_{1}(a)\right)$

Thus the solution in the first approximation is given by $\eta=a \cos (v+\theta)$, where the amplitude $a$ and phase $\theta$ are the solutions of the equations:

$\frac{d a}{d v}=\frac{-4 e}{n+1} \cos \theta, \quad \frac{d \theta}{d v}=(n-1)+\frac{4 e \sin \theta}{a(n+1)}-\frac{n}{2 a}\left(a-2 J_{1}(a)\right)$

Equations (4) cannot be integrated in a closed form due to dependence of the right hand side on $a$ and $\theta$. However, qualitative aspects of the solution can be examined. Taking $n \cong n_{e}=n\left(1-\frac{a^{2}}{16}\right)$ and $n=1$, eliminating the phase $\theta$, from the stationary regimes, we get, $n_{e}^{2}-1=-\frac{4 e}{a}$, Putting $\quad 1=n+\delta,(\delta<<1), \quad$ we obtain, $\delta=-n \pm \frac{1}{2 \sqrt{2}} \sqrt{\frac{32 e}{a}-n^{2}\left(a^{2}-8\right)}$. For instability (jump and fall), $\frac{d \delta}{d a}=0 \Rightarrow 8 \delta^{2}+16 n \delta+3 a^{2} n^{2}=0$. The two values of $\delta$ represented by the equation are both negative, so that the effect occurs only at a frequency of external periodic force which is less than the frequency of the natural oscillation of the system. Maximum value of the amplitude is obtained by the condition $\frac{d a}{d \delta}=0$, which gives $16 a(\delta+n)=0$.

\section{CONCLUSION}

The non-linear rotational equations of motion of the planar oscillation of a satellite in an elliptic orbit under the influence of magnetic torque are nonintegrable, as Melnikov's function has simple zeros. The amplitude of the oscillation remains constant up to the second order of approximation. The main resonances occur at $n \cong b$ and $n \cong 1$ and the parametric resonance appears only for $n \cong \frac{1}{2}$. The stability of the stationary planar oscillation of the satellite near the resonance frequency shows that discontinuity occurs in the amplitude of the oscillation at a frequency of the external periodic force which is less than the frequency of the natural oscillation. The jump in the amplitude at the critical point in the resonance curves increases with the increase in the magnetic torque and the eccentricity. 


\section{REFERENCES}

1. Maciejewski, A.J., 2001. Non-integrability of a certain problem of rotational motion of a rigid satellite. Eds., Prętka-Ziomek, Richardson. Dynamics of Natural and Artificial Celestial Bodies. Kluwer Academic Publisher, pp: 187-192.

2. Bhardwaj, R. and R. Tuli, 2005. Non-linear planar oscillation of a satellite leading to chaos under the influence of third-body torque. Mathematical models and Methods for real world systems. Eds., Nashed, Siddiqi. Chapman \& Hall/ CRC Publication, pp: 301-336.

3. Schweighart, S.A. and R.J. Sedwick, 2001. A perturbative analysis of geopotential disturbances for satellite formation flying. Proc. IEEE Aerospace Conf., 2: 1001-1019.

4. Suli, A., 2001. Structure of the outer 1:2 resonance in the Kuiper Belt. Publications of the Astronomy Department of the Eotvos University No. 11, Proceedings of the National Postgraduate Reunion in Astronomy \& Astrophysics, 35.
5. Michtchenko, T.A. and S. Ferraz-Mello, 2001. Resonant structure of the outer solar system in the neighborhood of the planets. The Astronomical J., 122: 474-481.

6. Murray, N. and M. Holman, 2001. The role of chaotic Resonance's in the Solar System. Nature, 410: 773-779.

7. Kotoulas, T.A. and J.D. Hadjidemetriou, 2002. Resonant periodic orbits of trans-neptunian objects. Earth, Moon and Planets, 9: 63-93.

8. Hadjidemetriou, J.D., 2002. Resonant periodic motion and the stability of extrasolar planetary systems. Celestial Mechanics and Dynamical Astronomy, 83: 141-154. 